\title{
Mutual Information of Amplify-and-Forward Relaying with Partial Relay Selection
}

\author{
Qiang Xue and Giuseppe Abreu \\ Centre for Wireless Communications \\ University of Oulu, Oulu, Finland \\ P.O.Box 4500 FIN-90014 \\ Email: xueqiang@ee.oulu.fi; giuseppe@ee.oulu.fi
}

\begin{abstract}
In this paper, we provide asymptotic analysis of the mutual information (MI) of the basic two-hop amplify-andforward (AF) channel, where the relaying node is selected out of a pool of $N$ potential relay nodes based on instantaneous backward channel state information (CSI). The asymptotic formula obtained by implementing extreme value theory (EVT) adopts a simpler form compared to the exact MI expression, which makes it easier to operate with. Despite its simplicity, the asymptotic expression is quite accurate even with small $N$, which is confirmed by numerical simulation. We show that the power allocation coefficient obtained by optimizing the asymptotic expression is close to the optimal value obtained from simulation results.
\end{abstract}

\section{INTRODUCTION}

The multi-relay channel similar to that illustrated in figure 1 have attracted much attention recently. In [1], for instance, a scheme was considered in which the transmitter, the relays and the receiver all have perfect and full (backward and forward) information of their own and each other's channel states. Assuming such a full CSI relay channel and that the source and each relay can transmit with any power within independent ranges $\left[0, P_{\max _{i}}\right]$ where $i$ denotes either the source or one of the relays, the authors derived the power allocation that maximizes the receive SNR for each channel realization and demonstrated that the optimal strategy is network beam-forming. In [2], an opportunistic relaying scheme is proposed where the best relay is selected from all the relay nodes based on their own forward and backward CSI. The selection is done in a distributed way. It is pointed out by [3] that although gaining both backward and forward CSI makes the transmission faster and reliable, there could be synchronization problems for a 2-link based distributed relay selection scheme because of its time sensitivity; and for a 2-link based centralized relay selection scheme, the demanding for continuous feedback results in an extra amount of power consumption. So for a cheap sensor network where power consumption overweights reliability in importance, it would be more interesting to consider relaying schemes that are simpler and yet perform well enough with less overhead signaling. Relaying schemes that implement distributed space-time block coding (DSTBC) belong to this category, because for DSTBC to work it is enough to gain only backward CSI, which means feedback is not needed and the active relays could be automatically selected. DSTBC schemes have been extensively studied, for instance, in [4], [5], [6],etc.

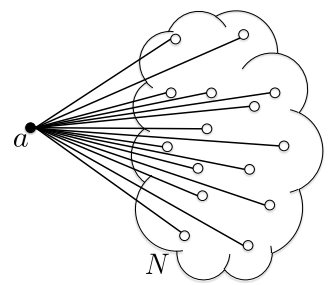

(a) Stage 1 .

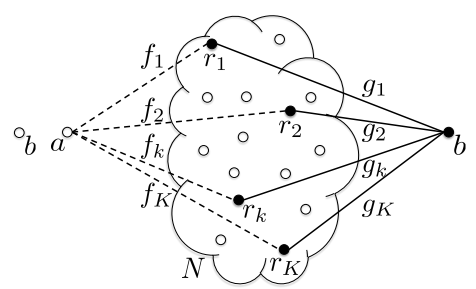

(b) Stage 2 .
Fig. 1. Illustration of the general system concept. Black and white dots represent active and inactive (non-transmitting) nodes, respectively. Specifically for our model, there will always be only 1 black dot for each transmission.

In this paper, we consider such a relaying scheme that the best node is selected out of a pool of $N$ potential nodes to amplify and forward the received information to the destination based on only their instantaneous backward channel state. The selection is done in a distributed way as in [2].

we analyze MI of the resulting channel. As will be seen from the following section, for each channel realization the channel is equivalent to an additive white gaussian noise (AWGN) channel whose capacity is well known. In [7], it was shown that for a fading channel if the channel sequence is i.i.d. and the input distribution which maximizes mutual information is the same regardless of the channel state, the capacity of the channel with CSI only at the receiver is given by the expectation of capacity of each channel realization. The constraint is satisfied in this scenario, so we derive the MI formula by taking the expectation of the AWGN capacity with respect to all channel states. We derive the exact expression of the MI which is in an integral form and difficult to operate. We then provide asymptotic analysis of the MI based on extreme value theory. The asymptotic analysis leads to a simpler formula which is easy to operate with, and its accuracy is confirmed by numerical simulation.

The remainder of this paper is organized as follows. Section II describes the system model. MI derivation and the asymptotic analysis is done in section III. Results and discussion are given in section IV. The final section is our main conclusion.

\section{System Model}

Consider a wireless network as illustrated in figure 1 where a source node denoted by $a$ communicates with a destination 
node denoted by $b$ through the help of a pool of $N$ relays, with no direct link between $a$ and $b$. Without sacrificing the essence of the results that will follow, we assume that the network is composed exclusively of single-antenna nodes.

For such a network, consider a two-stage amplify-andforward relaying mechanism where in the first stage, the source broadcasts a symbol vector $s$ to all $N$ relays, each of which will estimate the channel between the source and itself while receiving the signals. in the second stage, the relay node with the best backward channel is automatically selected ${ }^{1}$ to amplify and forward the source's signals to the destination.

Assume block fading and let the instantaneous complexvalued coefficients of the block-fading channels from $a$ to $r_{k}$ and from $r_{k}$ to $b$ be respectively denoted by $f_{k}$ and $g_{k}$; assume the coefficients to be independent and identically distributed (i.i.d.) with Rayleigh distribution and $\mathrm{E}\left[\left|f_{k}\right|^{2}\right]=\mathrm{E}\left[\left|g_{k}\right|^{2}\right]=1$. For each channel realization, denote the fading coefficient from source to the relaying node as $f_{m}$, and the fading coefficient from the relaying node to destination simply as $g$. So it is obvious that $\left|f_{m}\right|^{2}=\max _{i \in \mathcal{N}}\left|f_{i}\right|^{2}$ where $\mathcal{N}$ is the index set $\{1,2, \cdots, N\}$ and $|g|^{2}$ is an exponential random variable with unit rate.

Assume that the noise at all $r_{k}$ 's and $b$ are independent and identically distributed (i.i.d.) zero-mean complex-valued Gaussian variates with variance $\sigma^{2}$, and let $P_{1}$ denote the average transmit power of the source and $P_{2}$ as the average transmit power at the relay.

After stage 1, the received signal at the best relay is

$$
\boldsymbol{y}_{r}=\sqrt{P_{1}} f_{m} \boldsymbol{s}+w_{r} .
$$

where $w_{r}$ is the noise at the relay. Then after the relaying node amplifies the received signal $\boldsymbol{y}_{r}$ with a constant $\rho$ and forward it to the destination, the received signal at the destination is

$$
\boldsymbol{y}_{d}=\rho \sqrt{P_{1}} f_{m} g s+\rho g w_{r}+w_{d}
$$

where $w_{d}$ is the noise at the destination.

Notice that in equation (2) the amplification coefficient $\rho$ is assumed to be a real constant identical for all relays due to the symmetry of the relay channel and the fact that each relay is assumed to possess only its own backward CSI.

To satisfy the condition that the relaying node transmits at average power $P_{2}, \rho$ must be

$$
\rho=\sqrt{\frac{P_{2}}{\mathrm{E}\left[\left|f_{m}\right|^{2}\right] P_{1}+\sigma^{2}}} .
$$

As is shown above, $\left|f_{m}\right|^{2}$ is the maximum among $\mathrm{N}$ i.i.d exponential random variables with unit rate, so the probability density function (pdf) of $\left|f_{m}\right|^{2}$ is,

$$
p_{|f m|^{2}}(x)= \begin{cases}N e^{-x}\left(1-e^{-x}\right)^{N-1} & \text { if } z \geq 0 \\ 0 & \text { if } z<0\end{cases}
$$

\footnotetext{
${ }^{1}$ We adopt the relay selection scheme proposed in [2], which is fast and energy efficient since it is a decentralized relay selection protocol. We omit the detailed description and analysis of the protocol here and refer them to the references of [2].
}

So the expectation ${ }^{2}$ of $\left|f_{m}\right|^{2}$ is given by,

$$
\begin{aligned}
\mathrm{E}\left[\left|f_{m}\right|^{2}\right] & =\int_{0}^{\infty} x N e^{-x}\left(1-e^{-x}\right)^{N-1} \mathrm{~d} x \\
& =\sum_{i=1}^{N}(-1)^{i+1}\left(\begin{array}{c}
N \\
i
\end{array}\right) \frac{1}{i} .
\end{aligned}
$$

Which is only dependent on $N$.

As can be seen from equation (2), for each channel realization, the channel is equivalent to an AWGN channel, whose signal to noise ratio denoted by $\gamma_{a b}$ is given by,

$$
\gamma_{a b}=\frac{\rho^{2} P_{1}\left|f_{m}\right|^{2}|g|^{2}}{\sigma^{2}+\rho^{2}|g|^{2} \sigma^{2}}
$$

\section{Mutual Information AnAlysis}

It is well known that the capacity of an AWGN channel with complex codebook is given by [8]

$$
C=\log (1+\mathrm{SNR}) \quad \text { bit/dimension pair. }
$$

Assuming perfect interleaving the channel can be treated as an ergodic fading channel, whose average MI is shown in [9] to be the expectation of the capacity of each channel realization. Denote $\mathcal{I}$ as the average MI of the channel. Then,

$$
\mathcal{I}=\mathrm{E}\left[\log \left(1+\gamma_{a b}\right)\right] .
$$

Substituting equation (6) in to equation (8), and by denoting $\gamma_{1}=P_{1} / \sigma^{2},|g|^{2}=x,\left|f_{m}\right|^{2}=y$ yields,

$$
\mathcal{I}=\int_{0}^{\infty} e^{-x} \mathrm{~d} x \int_{0}^{\infty} \log \left(1+\gamma_{1} \frac{x}{x+\rho^{-2}} \cdot y\right) p_{|f m|^{2}}(y) \mathrm{d} y
$$

There are two ways to simplify the integral. The first way is to do exact derivation: Substituting equation (4) into (9) yields,

$$
\mathcal{I}=\int_{0}^{\infty} \int_{0}^{\infty} \log \left(1+\gamma_{1} \frac{x y}{x+\rho^{-2}}\right) e^{-x} N e^{-y}\left(1-e^{-y}\right)^{N-1} \mathrm{~d} x \mathrm{~d} y
$$

After some manipulation ${ }^{3}$ we have,

$$
\begin{aligned}
\mathcal{I}= & \frac{1}{\ln 2} \sum_{k=0}^{N-1}(-1)^{k}\left(\begin{array}{c}
N \\
k+1
\end{array}\right) \exp \left(\frac{k+1}{\gamma_{1}}\right) \times \\
& \times \int_{0}^{\infty} \mathrm{E}_{1}\left[\frac{(k+1)\left(x+\rho^{-2}\right)}{\gamma_{1} x}\right] \exp \left(\frac{\rho^{-2}(k+1)-\gamma_{1} x^{2}}{\gamma_{1} x}\right) \mathrm{d} x
\end{aligned}
$$

where $\mathrm{E}_{1}(\cdot)$ denotes the $\mathrm{E}_{n}$-function with $n=1$ whose definition can be found in [12].

Notice that the exact expression (11) contains an infinite integral of the exponential integral function, which makes it

\footnotetext{
${ }^{2}$ detailed derivation can be found in the appendix

${ }^{3}$ which is omitted here and can be found in the appendix
} 
difficult to operate with. And it is nearly impossible to get an exact expression with other statistical fading models. As we can see in the following part, the second way, which is to do asymptotic analysis, generates simpler expression without sacrificing much accuracy. It could also be generalized to more complex fading models like the Nakagami.

The asymptotic analysis is based on the extreme value theory, which is similar to central limit theory. Both of them deal with asymptotic distribution of random variables. Specifically, Let random variables $\alpha_{1}, \alpha_{2}, \cdots, \alpha_{M}$ be generated independently according to the same cumulative distribution function (CDF) $F(x)$. Define $A_{M} \triangleq \max _{i \in \mathcal{M}} \alpha_{i}$ where $\mathcal{M}$ is the index set $\{1,2, \cdots, M\}$. Then in general, for a regularly behaved $F(x)$, there exist constants $a_{M}$ and $b_{M}$, such that the distribution of $\left(A_{M}-a_{m}\right) / b_{M}$ converges to one of the three standard extreme value distributions: Gumbel, Frechet and Webull distributions [10]. It is proved in [10] that when $F(x)$ is CDF of exponential distribution, $\left(A_{M}-a_{m}\right) / b_{M}$ converges to Gumbel distribution.

In this paper, we are interested in the asymptotic expectation of a function of the maximum random variable $A_{M}$, which is $\mathrm{E}\left[\log \left(1+c A_{M}\right)\right]$ where $c$ is a constant. It is proved in [11] that when $F(x)$ is CDF of exponential distribution with unit rate parameter, the asymptotic expectation adopts a simple form as:

$\mathrm{E}\left[\log \left(1+c A_{M}\right)\right] \approx \log (1+c \ln M)+E_{0} \log \left(1+\frac{c}{1+c \ln M}\right)$

where $E_{0}$ is the euler constant. Notice that the inner integral in equation (9) has exactly the same form, so,

$$
\begin{aligned}
\mathcal{I} & =\int_{0}^{\infty} e^{-x} \mathrm{~d} x \mathrm{E}_{y}\left[\log \left(1+\gamma_{1} \frac{x}{x+\rho^{-2}} \cdot y\right)\right] \\
= & \int_{0}^{\infty} \log \left(1+\gamma_{1} \frac{x}{x+\rho^{-2}} \ln N\right) e^{-x} \mathrm{~d} x \\
& +E_{0} \int_{0}^{\infty} \log \left(1+\frac{\gamma_{1} \frac{x}{x+\rho^{-2}}}{\gamma_{1} \frac{x}{x+\rho^{-2}} \ln N}\right) e^{-x} \mathrm{~d} x
\end{aligned}
$$

(13) could be simplied as ${ }^{4}$,

$\mathcal{I}=\frac{1}{\ln 2}\left(1-E_{0}\right) \exp \left[\frac{1}{\left(\gamma_{1} \ln N+1\right) \rho^{2}}\right] \mathrm{E}_{1}\left[\frac{1}{\left(\gamma_{1} \ln N+1\right) \rho^{2}}\right]$ $+\frac{1}{\ln 2} E_{0} \exp \left[\frac{1}{\left(\gamma_{1} \ln N+\gamma_{1}+1\right) \rho^{2}}\right] \mathrm{E}_{1}\left[\frac{1}{\left(\gamma_{1} \ln N+\gamma_{1}+1\right) \rho^{2}}\right]$ $-\frac{1}{\ln 2} \exp \left(\frac{1}{\rho^{2}}\right) \mathrm{E}_{1}\left(\frac{1}{\rho^{2}}\right)$

\section{RESULTS}

First, we examine the accuracy of the asymptotic formula and the exact formula by comparing the MI computed from them with the MI obtained by running Monte Carlo simulation.

\footnotetext{
${ }^{4}$ again, the derivation is omitted here, and it can be found in the appendix
}

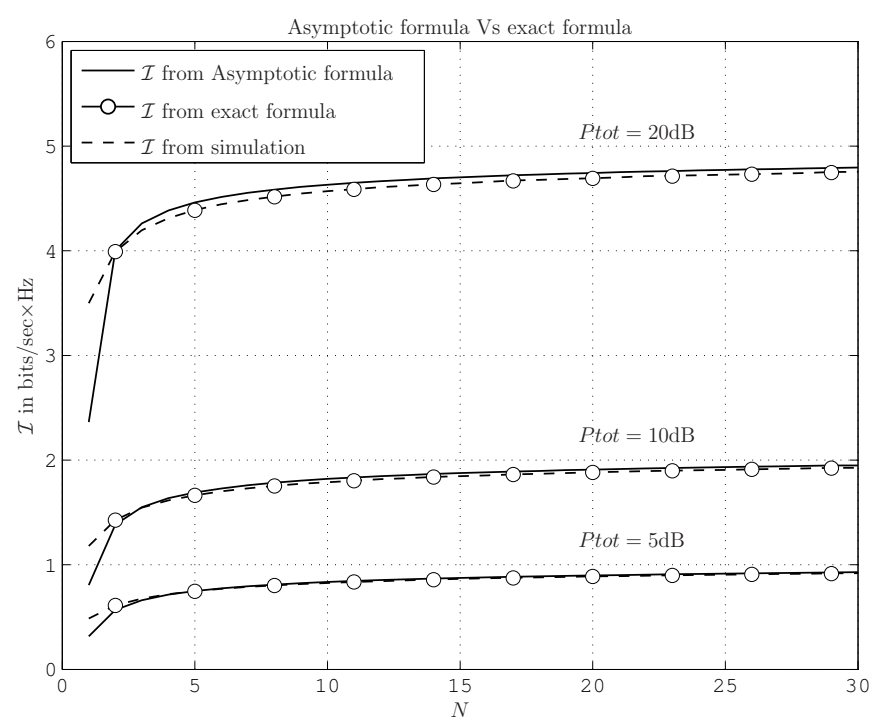

Fig. 2. Performance of the asymptotic formula and exact formula.

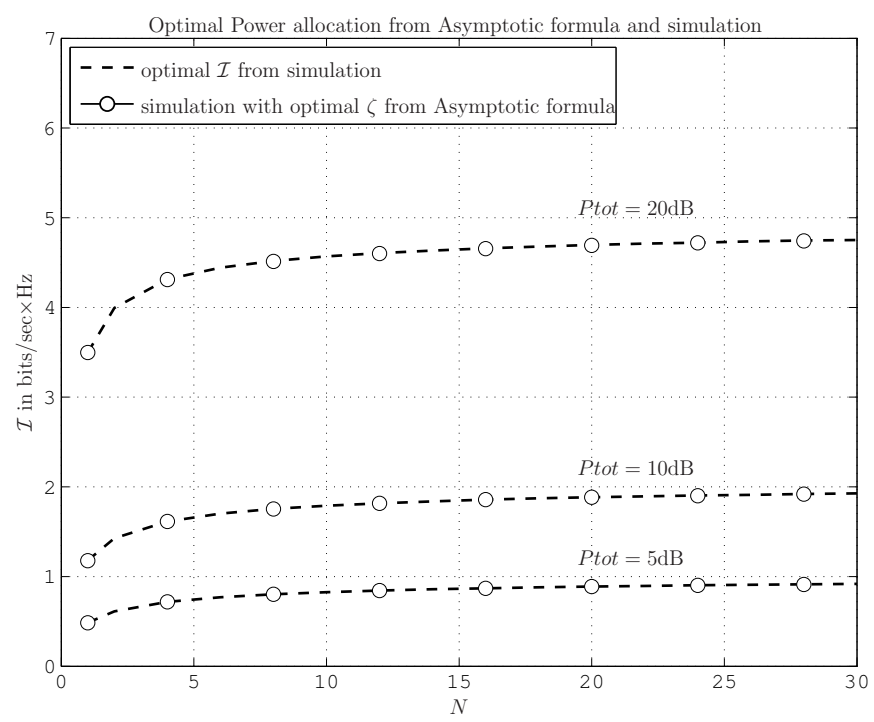

Fig. 3. The optimal power allocation coefficient obtained from asymptotic analysis fits well with the global optimal values.

Instead of using $P_{1}$ and $P_{2}$, we introduce another pair of parameters to represent the power allocation between the 2 transmission stages, which are defined as Ptot $\triangleq P_{1}+P_{2}$, $\zeta \triangleq P_{1} /$ Ptot. We consider the cases that Ptot takes the values of $5 \mathrm{~dB}, 10 \mathrm{~dB}, 20 \mathrm{~dB}$ and $N$ is from 1 to 30 . We first run the Monte Carlo simulation for each setting point and find the optimal $\zeta$. Then by calculating MI corresponding to the Ptot and $\zeta$ from the asymptotic formula and exact formula separately, we obtain figure 2 . From figure 2 it is observed that despite the simplicity of the asymptotic formula, it is very accurate even when $\mathrm{N}$ is small. Since the asymptotic formula is tight to the simulation, it is interesting to check whether we can get the optimal parameter setting for the system from it such 
that we could avoid operating on the complex exact formula. We consider the same cases that Ptot takes the values of $5 \mathrm{~dB}, 10 \mathrm{~dB}, 20 \mathrm{~dB}$ and $N$ is from 1 to 30 . For each setting point, we obtain the optimal $\zeta$ by optimizing the asymptotic formula, then we do simulation by implementing these parameters, and finally by comparing the result with the optimal MI obtained from pure simulation, we get figure 3 . It is observed that the parameter obtained from optimizing the asymptotic formula fits very well with the global optimal parameter.

\section{CONCLUSION}

we provide asymptotic analysis of the mutual information (MI) of the basic two-hop amplify-and-forward (AF) channel with partial relay selection. We find that the asymptotic formula is powerful not only because of its simplicity and accuracy but also because of its validity as a tool to find the optimal working point for such a system.

\section{APPENDIX \\ A. Derivation of $\mathrm{E}\left[\left|f_{m}\right|^{2}\right]$}

$$
\begin{aligned}
\mathrm{E}\left[\left|f_{m}\right|^{2}\right] & =\int_{0}^{\infty} x N e^{-x}\left(1-e^{-x}\right)^{N-1} \mathrm{~d} x \\
& =N \int_{0}^{\infty} x e^{-x} \sum_{k=0}^{N-1}\left(\begin{array}{c}
N-1 \\
k
\end{array}\right)\left(-e^{-x}\right)^{k} \mathrm{~d} x \\
& =N \sum_{k=0}^{N-1}(-1)^{k}\left(\begin{array}{c}
N-1 \\
k
\end{array}\right) \int_{0}^{\infty} x e^{-(k+1) x} \mathrm{~d} x \\
& =N \sum_{k=0}^{N-1}(-1)^{k}\left(\begin{array}{c}
N-1 \\
k
\end{array}\right) \frac{1}{(k+1)^{2}} \\
& =\sum_{i=1}^{N}(-1)^{i+1}\left(\begin{array}{c}
N \\
i
\end{array}\right) \frac{1}{i} .
\end{aligned}
$$

\section{B. Derivation of exact $M I$}

$$
\begin{aligned}
\mathcal{I} & =\int_{0}^{\infty} \int_{0}^{\infty} \log \left(1+\gamma_{1} \frac{x y}{x+\rho^{-2}}\right) e^{-x} N e^{-y}\left(1-e^{-y}\right)^{N-1} \mathrm{~d} x \mathrm{~d} y \\
& =N \sum_{k=0}^{N-1}\left(\begin{array}{c}
N-1 \\
k
\end{array}\right)(-1)^{k} \int_{0}^{\infty} \int_{0}^{\infty} \log \left(1+\frac{\gamma_{1} x y}{x+\rho^{-2}}\right) e^{-x-(k+1) y} \mathrm{~d} x \mathrm{~d} y
\end{aligned}
$$

According to [12, pp.341, Eq. 3.353.5], by integrating with respect to $y$, equation (16) becomes,

$$
\begin{aligned}
\mathcal{I}= & \frac{1}{\ln 2} \sum_{k=0}^{N-1}(-1)^{k}\left(\begin{array}{c}
N \\
k+1
\end{array}\right) \exp \left(\frac{k+1}{\gamma_{1}}\right) \times \\
& \times \int_{0}^{\infty} \mathrm{E}_{1}\left[\frac{(k+1)\left(x+\rho^{-2}\right)}{\gamma_{1} x}\right] \exp \left(\frac{\rho^{-2}(k+1)-\gamma_{1} x^{2}}{\gamma_{1} x}\right) \mathrm{d} x
\end{aligned}
$$

where $\mathrm{E}_{1}(\cdot)$ denotes the $\mathrm{E}_{n}$-function with $n=1$.

\section{Derivation of EVT MI}

According to [12, pp.572, Eq. 4.337.2],

$$
\begin{aligned}
& \int_{0}^{\infty} \log \left(1+\gamma_{1} \frac{x}{x+\rho^{-2}} \ln N\right) e^{-x} \mathrm{~d} x= \\
& -\frac{1}{\ln 2} \exp \left[\frac{1}{\left(\gamma_{1} \ln N+1\right) \rho^{2}}\right] \mathrm{E}_{i}\left[\frac{-1}{\left(\gamma_{1} \ln N+1\right) \rho^{2}}\right] \\
& +\frac{1}{\ln 2} \exp \left(\frac{1}{\rho^{2}}\right) \mathrm{E}_{i}\left(\frac{-1}{\rho^{2}}\right)
\end{aligned}
$$

$$
\begin{aligned}
& \int_{0}^{\infty} \log \left(1+\frac{\gamma_{1} \frac{x}{x+\rho^{-2}}}{\gamma_{1} \frac{x}{x+\rho^{-2}} \ln N}\right) e^{-x} \mathrm{~d} x= \\
& \frac{1}{\ln 2} \exp \left[\frac{1}{\left(\gamma_{1} \ln N+1\right) \rho^{2}}\right] \mathrm{E}_{i}\left[\frac{-1}{\left(\gamma_{1} \ln N+1\right) \rho^{2}}\right]-\frac{1}{\ln 2} \\
& \times \exp \left[\frac{1}{\left(\gamma_{1} \ln N+\gamma_{1}+1\right) \rho^{2}}\right] \mathrm{E}_{i}\left[\frac{-1}{\left(\gamma_{1} \ln N+\gamma_{1}+1\right) \rho^{2}}\right]
\end{aligned}
$$

Where $\mathrm{E}_{i}(\cdot)$ is the exponential integral function. Notice that $\mathrm{E}_{i}(x)=-\mathrm{E}_{1}(x)$, and substitute equations (18) and (19) in to (13), then

$$
\begin{aligned}
\mathcal{I} & =\frac{1}{\ln 2}\left(1-E_{0}\right) \exp \left[\frac{1}{\left(\gamma_{1} \ln N+1\right) \rho^{2}}\right] \mathrm{E}_{1}\left[\frac{1}{\left(\gamma_{1} \ln N+1\right) \rho^{2}}\right] \\
& +\frac{1}{\ln 2} E_{0} \exp \left[\frac{1}{\left(\gamma_{1} \ln N+\gamma_{1}+1\right) \rho^{2}}\right] \mathrm{E}_{1}\left[\frac{1}{\left(\gamma_{1} \ln N+\gamma_{1}+1\right) \rho^{2}}\right] \\
& -\frac{1}{\ln 2} \exp \left(\frac{1}{\rho^{2}}\right) \mathrm{E}_{1}\left(\frac{1}{\rho^{2}}\right)
\end{aligned}
$$

\section{REFERENCES}

[1] Y. Jing and H. Jafarkhani, "Network beamforming using relays with perfect channel information," in Proc. IEEE Int. Conf. of Acoustics, Speech, and Sig. Proc.(ICASSP'07), Hawaii, USA, Apr. 2007.

[2] A. Bletsas, H. Shin, and M. Z. Win, "Cooperative communications with outage-optimal opportunistic relaying," IEEE Trans. Commun., vol. 6, no. 9, pp. 3450-3460, 2007.

[3] I. Krikidis, J. Thompson, S. McLaughlin, and N. Goertz, "Amplify-andforward with partial relay selection," IEEE Commun. Lett., vol. 12, no. 4, pp. 235-237, 2008.

[4] J. N. Laneman and G. W. Wornell, "Distributed space-time-coded protocols for exploiting cooperative diversity in wireless networks," IEEE Trans. Inf. Theory, vol. 49, no. 10, pp. 2415-2425, Oct. 2003.

[5] Y. Jing and B. Hassibi, "Distributed space-time coding in wireless relay networks," IEEE Trans. Wireless Commun., vol. 5, no. 12, pp. 3524 3536, Dec. 2006.

[6] B. Maham, A. Hjorungnes, and G. Abreu, "Distributed GABBA spacetime codes in amplify-and-forward relay networks," IEEE Transactions. Wireless Communications., vol. 8, no. 4, pp. 2036-2045, Apr 2009.

[7] R. J. McEliece and W. E. Stark, "Channels with block interference," IEEE Trans. Inf. Theory, vol. IT-30, pp. 44-53, Jan. 1984.

[8] E. Biglieri, Coding for wireless channels, 1st ed. Springer, 2005.

[9] A. J. Goldsmith and P. P. Varaiya, "Capacity of fading channels with channel side information," IEEE Trans. Inf. Theory, vol. 43, no. 9, pp. 1986-1992, Nov. 1997.

[10] H.A.David, Order Statistics. New York: Wiley, 1970

[11] G. Song and Y. Li, "Asymptotic throughput analysis for channel-aware scheduling," IEEE Trans. Commun., vol. 54, no. 10, pp. 1827-1833, 2006.

[12] I. S. Gradshteyn and I. M. Ryzhik, Table of Integrals, Series, and Products, 7th ed. Academic Press, 2007. 